\title{
On the Solutions of Fractional Burgers-Fisher and Generalized Fisher's Equations Using Two Reliable Methods
}

\author{
A. K. Gupta and S. Saha Ray \\ Department of Mathematics, National Institute of Technology, Rourkela 769008, India \\ Correspondence should be addressed to S. Saha Ray; santanusaharay@yahoo.com
}

Received 7 January 2014; Revised 25 March 2014; Accepted 25 March 2014; Published 8 May 2014

Academic Editor: Shyam L. Kalla

Copyright ( 2014 A. K. Gupta and S. S. Ray. This is an open access article distributed under the Creative Commons Attribution License, which permits unrestricted use, distribution, and reproduction in any medium, provided the original work is properly cited.

Two reliable techniques, Haar wavelet method and optimal homotopy asymptotic method (OHAM), are presented. Haar wavelet method is an efficient numerical method for the numerical solution of arbitrary order partial differential equations like BurgersFisher and generalized Fisher equations. The approximate solutions thus obtained for the fractional Burgers-Fisher and generalized Fisher equations are compared with the optimal homotopy asymptotic method as well as with the exact solutions. Comparison between the obtained solutions with the exact solutions exhibits that both the featured methods are effective and efficient in solving nonlinear problems. The obtained results justify the applicability of the proposed methods for fractional order Burgers-Fisher and generalized Fisher's equations.

\section{Introduction}

Fractional calculus is a field of applied mathematics which deals with derivatives and integrals of arbitrary orders. In the last few decades, fractional calculus has been extensively investigated due to its broad applications in mathematics, physics, and engineering such as viscoelasticity, diffusion of biological population, signal processing, electromagnetism, fluid mechanics, electrochemistry and so on. Fractional differential equations are extensively used in modeling of physical phenomena in various fields of science and engineering. For this we need a reliable and efficient technique for the solution of fractional differential equations.

Recently, orthogonal wavelets bases are becoming more popular for numerical solutions of partial differential equations due to their excellent properties such as ability to detect singularities, orthogonality, flexibility to represent a function at different levels of resolution, and compact support. In recent years, there has been a growing interest in developing wavelet based numerical algorithms for solution of fractional order partial differential equations. Among them, the Haar wavelet method is the simplest and is easy to use. Haar wavelets have been successfully applied for the solutions of ordinary and partial differential equations, integral equations, and integrodifferential equations. Therefore, the main focus of the present paper is the application of Haar wavelet technique for solving the problem of Burgers-Fisher and generalized Fisher's equations. The obtained numerical approximation results of this method are then also compared with the optimal homotopy asymptotic method.

Consider the generalized one-dimensional BurgersFisher equation of fractional order:

$$
\frac{\partial^{\alpha} u}{\partial t^{\alpha}}+\xi u^{\eta} \frac{\partial u}{\partial x}=\mu \frac{\partial^{2} u}{\partial x^{2}}+\beta u\left(1-u^{\eta}\right)
$$

where $\xi$, $\mu$, and $\beta$ are parameters and $0<\alpha \leq 1$. This equation has a wide range of applications in fluid dynamics model, heat conduction, elasticity, and capillary-gravity waves. When $\xi=$ 0 and $\eta=1$, (1) reduces to Fisher type equation. The derivative in (1) is Caputo derivative of order $\alpha$.

The generalized time-fractional Fisher's biological population diffusion equation is given by

$$
\frac{\partial^{\alpha} u}{\partial t^{\alpha}}=\frac{\partial^{2} u}{\partial x^{2}}+F(u), \quad u(x, 0)=\varphi(x),
$$

where $u(x, t)$ denotes the population density and $t>0, x \in$ $\mathfrak{R}$, and $F(u)$ is a continuous nonlinear function satisfying 
the following conditions: $F(0)=F(1)=0, F^{\prime}(0)>0>$ $F^{\prime}(1)$. The derivative in (2) is also the Caputo derivative of order $\alpha$.

Our aim in the present work is to implement Haar wavelet method and optimal homotopy asymptotic method (OHAM) in order to demonstrate the capability of these methods in handling nonlinear equations of arbitrary order, so that one can apply it to various types of nonlinearity.

\section{Fractional Derivative and Integration}

There are several approaches to define the derivatives of fractional order such as Riemann-Liouville, Grünwald-Letnikov, and Caputo. Riemann-Liouville fractional derivative is not suitable for real-world physical problems since it requires the definition of fractional order initial conditions, which have no physically meaningful explanation yet. Caputo introduced an alternative definition, which has the advantage of defining integer order initial conditions for fractional order differential equations.

Definition 1. The Riemann-Liouville fractional integral operator $J^{\alpha}(\alpha \geq 0)$, of a function $f(t)$, is defined as [1]

$$
J^{\alpha} f(t)=\frac{1}{\Gamma(\alpha)} \int_{0}^{t}(t-\tau)^{\alpha-1} f(\tau) d \tau, \quad \alpha>0, \alpha \in \mathfrak{R}^{+},
$$

where $\Gamma(\cdot)$ is the well-known gamma function, and some properties of the operator $J^{\alpha}$ are as follows:

$$
\begin{gathered}
J^{\alpha} J^{\beta} f(t)=J^{\alpha+\beta} f(t), \quad(\alpha>0, \beta>0), \\
J^{\alpha} t^{\gamma}=\frac{\Gamma(1+\gamma)}{\Gamma(1+\gamma+\alpha)} t^{\alpha+\gamma}, \quad(\gamma>-1) .
\end{gathered}
$$

Definition 2. The Caputo fractional derivative ${ }_{0} D_{t}^{\alpha}$ of a function $f(t)$ is defined as [1]

$$
\begin{array}{r}
{ }_{0} D_{t}^{\alpha} f(t)=\frac{1}{\Gamma(n-\alpha)} \int_{0}^{t} \frac{f^{n}(\tau)}{(t-\tau)^{\alpha-n+1}} d \tau, \\
\quad(n-1<\alpha \leq n, n \in N) .
\end{array}
$$

The following are two basic properties of the Caputo fractional derivative:

$$
\begin{gathered}
{ }_{0} D_{t}^{\alpha} t^{\beta}=\frac{\Gamma(1+\beta)}{\Gamma(1+\beta-\alpha)} t^{\beta-\alpha}, \quad 0<\alpha<\beta+1, \beta>-1, \\
J^{\alpha} D^{\alpha} f(t)=f(t)-\sum_{k=0}^{n-1} f^{k}\left(0^{+}\right) \frac{t^{k}}{k !} \\
n-1<\alpha \leq n, n \in N .
\end{gathered}
$$

\section{Haar Wavelets}

Haar functions have been used from 1910 when they were introduced by the Hungarian mathematician Alfred Haar.
Haar wavelets are the simplest wavelets among various types of wavelets. They are step functions over the real line that can take only three values 0,1 , and -1 . The method has been used for being its simpler, fast, and computationally attractive feature. Usually the Haar wavelets are defined for the interval $t \in[0,1)$ but in general case $t \in[A, B]$, we divide the interval $[A, B]$ into $m$ equal subintervals, each of width $\Delta t=$ $(B-A) / m$. In this case, the orthogonal set of Haar functions are defined in the interval $[A, B]$ by $[2]$

$$
\begin{gathered}
h_{0}(t)= \begin{cases}1 & t \in[A, B], \\
0 & \text { elsewhere, }\end{cases} \\
h_{i}(t)= \begin{cases}1, & \zeta_{1}(i) \leq t<\zeta_{2}(i) \\
-1, & \zeta_{2}(i) \leq t<\zeta_{3}(i) \\
0, & \text { otherwise, }\end{cases}
\end{gathered}
$$

where

$$
\begin{gathered}
\zeta_{1}(i)=A+\left(\frac{k-1}{2^{j}}\right)(B-A)=A+\left(\frac{k-1}{2^{j}}\right) m \Delta t, \\
\zeta_{2}(i)=A+\left(\frac{k-(1 / 2)}{2^{j}}\right)(B-A)=A+\left(\frac{k-(1 / 2)}{2^{j}}\right) m \Delta t, \\
\zeta_{3}(i)=A+\left(\frac{k}{2^{j}}\right)(B-A)=A+\left(\frac{k}{2^{j}}\right) m \Delta t,
\end{gathered}
$$

$i=1,2, \ldots, m, m=2^{J}$, and $J$ is a positive integer which is called the maximum level of resolution. Here $j$ and $k$ represent the integer decomposition of the index $i$. That is, $i=k+2^{j}-1,0 \leq j<i$, and $1 \leq k<2^{j}+1$.

\section{Function Approximation}

Any function $y(t) \in L^{2}([0,1))$ can be expanded into Haar wavelets by $[2-4]$

$$
\begin{array}{r}
y(t)=c_{0} h_{0}(t)+c_{1} h_{1}(t)+c_{2} h_{2}(t)+\cdots, \\
\text { where } c_{j}=\int_{0}^{1} y(t) h_{j}(t) d t .
\end{array}
$$

If $y(t)$ is approximated as piecewise constant in each subinterval, the sum in (9) may be terminated after $m$ terms and consequently we can write discrete version in the matrix form as

$$
\mathbf{Y} \approx \sum_{i=0}^{m-1} c_{i} h_{i}\left(t_{l}\right)=\mathbf{C}_{\mathbf{m}}^{\mathbf{T}} H_{m}
$$

where $\mathbf{Y}$ and $\mathbf{C}_{\mathbf{m}}^{\mathbf{T}}$ are the $m$-dimensional row vectors.

Here $H$ is the Haar wavelet matrix of order $m$ defined by $H=\left[\mathbf{h}_{\mathbf{0}}, \mathbf{h}_{\mathbf{1}}, \ldots, \mathbf{h}_{\mathbf{m}-\mathbf{1}}\right]^{T}$; that is,

$$
H=\left[\begin{array}{c}
\mathbf{h}_{\mathbf{0}} \\
\mathbf{h}_{\mathbf{1}} \\
\vdots \\
\mathbf{h}_{\mathbf{m}-\mathbf{1}}
\end{array}\right]=\left[\begin{array}{cccc}
h_{0,0} & h_{0,1} & \cdots & h_{0, m-1} \\
h_{1,0} & h_{1,1} & \cdots & h_{1, m-1} \\
\vdots & & & \\
h_{m-1,0} & h_{m-1,1} & \cdots & h_{m-1, m-1}
\end{array}\right] \text {, }
$$


where $\mathbf{h}_{\mathbf{0}}, \mathbf{h}_{\mathbf{1}}, \ldots, \mathbf{h}_{\mathbf{m}-\mathbf{1}}$ are the discrete form of the Haar wavelet bases.

The collocation points are given by

$$
t_{l}=A+(l-0.5) \Delta t, \quad l=1,2, \ldots, m .
$$

\section{Operational Matrix of the General Order Integration [2]}

The integration of the $H_{m}(t)=\left[h_{0}(t), h_{1}(t), \ldots, h_{m-1}(t)\right]^{T}$ can be approximated by [4]

$$
\int_{0}^{t} H_{m}(\tau) d \tau \cong Q H_{m}(t)
$$

where $Q$ is called the Haar wavelet operational matrix of integration which is a square matrix of $m$-dimension. To derive the Haar wavelet operational matrix of the general order of integration, we recall the fractional integral of order $\alpha(>0)$ which is defined by Podlubny [1]

$$
J^{\alpha} f(t)=\frac{1}{\Gamma(\alpha)} \int_{0}^{t}(t-\tau)^{\alpha-1} f(\tau) d \tau, \quad t>0, \alpha \in \mathfrak{R}^{+},
$$

where $\mathfrak{R}^{+}$is the set of positive real numbers.

The Haar wavelet operational matrix $Q^{\alpha}$ for integration of the general order $\alpha$ is given by

$$
\begin{aligned}
Q^{\alpha} H_{m}(t) & =J^{\alpha} H_{m}(t)=\left[J^{\alpha} h_{0}(t), J^{\alpha} h_{1}(t), \ldots, J^{\alpha} h_{m-1}(t)\right]^{T} \\
& =\left[Q h_{0}(t), Q h_{1}(t), \ldots, Q h_{m-1}(t)\right]^{T},
\end{aligned}
$$

where

$$
\begin{gathered}
Q h_{0}(t)= \begin{cases}\frac{t^{\alpha}}{\Gamma(1+\alpha)}, & t \in[A, B], \\
0, & \text { elsewhere, }\end{cases} \\
Q h_{i}(t)= \begin{cases}0, & A \leq t<\zeta_{1}(i), \\
\phi_{1}, & \zeta_{1}(i) \leq t<\zeta_{2}(i), \\
\phi_{2}, & \zeta_{2}(i) \leq t<\zeta_{3}(i), \\
\phi_{3}, & \zeta_{3}(i) \leq t<B,\end{cases}
\end{gathered}
$$

where

$$
\begin{aligned}
& \phi_{1}=\frac{\left(t-\zeta_{1}(i)\right)^{\alpha}}{\Gamma(\alpha+1)} \\
& \phi_{2}=\frac{\left(t-\zeta_{1}(i)\right)^{\alpha}}{\Gamma(\alpha+1)}-2 \frac{\left(t-\zeta_{2}(i)\right)^{\alpha}}{\Gamma(\alpha+1)} \\
& \phi_{3}=\frac{\left(t-\zeta_{1}(i)\right)^{\alpha}}{\Gamma(\alpha+1)}-2 \frac{\left(t-\zeta_{2}(i)\right)^{\alpha}}{\Gamma(\alpha+1)}+\frac{\left(t-\zeta_{3}(i)\right)^{\alpha}}{\Gamma(\alpha+1)}
\end{aligned}
$$

for $i=1,2, \ldots, m, m=2^{J}$ and $J$ is a positive integer, called the maximum level of resolution. Here $j$ and $k$ represent the integer decomposition of the index $i$. That is, $i=k+2^{j}-1,0 \leq$ $j<i$, and $1 \leq k<2^{j}+1$.

\section{Basic Idea of Optimal Homotopy Asymptotic Method (OHAM)}

The OHAM was introduced and developed by Marinca et al. [5]. In OHAM, the control and adjustment of the convergence region are provided in a convenient way. To illustrate the basic ideas of optimal homotopy asymptotic method [6, 7], we consider the following nonlinear differential equation:

$$
A(u(x, t))+g(x, t)=0, \quad x \in \Omega
$$

with the boundary conditions

$$
B\left(u, \frac{\partial u}{\partial t}\right)=0, \quad x \in \Gamma
$$

where $A$ is a differential operator, $B$ is a boundary operator, $u(x, t)$ is an unknown function, $\Gamma$ is the boundary of the domain $\Omega$, and $g(x, t)$ is a known analytic function.

The operator $A$ can be decomposed as

$$
A=L+N \text {, }
$$

where $L$ is a linear operator and $N$ is a nonlinear operator.

We construct a homotopy $\varphi(x, t ; p): \Omega \times[0,1] \rightarrow \mathfrak{R}$ which satisfies

$$
\begin{aligned}
H(\varphi(x, t ; p), p)= & (1-p)[L(\varphi(x, t ; p))+g(x, t)] \\
& -H(p)[A(\varphi(x, t ; p))+g(x, t)]=0,
\end{aligned}
$$

where $p \in[0,1]$ is an embedding parameter, $H(p)$ is a nonzero auxiliary function for $p \neq 0$, and $H(0)=0$. When $p=0$ and $p=1$, we have $\varphi(x, t ; 0)=u_{0}(x, t)$ and $\varphi(x, t ; 1)=$ $u(x, t)$, respectively.

Thus as $p$ varies from 0 to 1 , the solution $\varphi(x, t ; p)$ approaches from $u_{0}(x, t)$ to $u(x, t)$.

Here $u_{0}(x, t)$ is obtained from (21) and (19) with $p=0$ yields

$$
L(\varphi(x, t ; 0))+g(x, t)=0, \quad B\left(u_{0}, \frac{\partial u_{0}}{\partial t}\right)=0
$$

The auxiliary function $H(p)$ is chosen in the form

$$
H(p)=p C_{1}+p^{2} C_{2}+p^{3} C_{3}+\cdots,
$$

where $C_{1}, C_{2}, C_{3}, \ldots$ are constants to be determined. To get an approximate solution, $\widetilde{\varphi}\left(x, t ; p, C_{1}, C_{2}, C_{3}, \ldots\right)$ is expanded in a series about $p$ as

$$
\begin{aligned}
\widetilde{\varphi}(x, t & \left.; p, C_{1}, C_{2}, C_{3}, \ldots\right) \\
& =u_{0}(x, t)+\sum_{i=1}^{\infty} u_{i}\left(x, t, C_{1}, C_{2}, C_{3}, \ldots\right) p^{i} .
\end{aligned}
$$


Substituting (24) in (21) and equating the coefficients of like powers of $p$, we will have the following equations:

$$
\begin{gathered}
L\left(u_{1}(x, t)+g(x, t)\right)=C_{1} N_{0}\left(u_{0}(x, t)\right), \\
B\left(u_{1}, \frac{\partial u_{1}}{\partial t}\right)=0, \\
L\left(u_{2}(x, t)\right)-L\left(u_{1}(x, t)\right) \\
=C_{2} N_{0}\left(u_{0}(x, t)\right) \\
+C_{1}\left(L\left(u_{1}(x, t)\right)+N_{1}\left(u_{0}(x, t), u_{1}(x, t)\right)\right), \\
B\left(u_{2}, \frac{\partial u_{2}}{\partial t}\right)=0 .
\end{gathered}
$$

And hence the general governing equations for $u_{j}(x, t)$ are given by

$$
\begin{aligned}
L\left(u_{j}(x, t)\right)=L\left(u_{j-1}(x, t)\right)+C_{j} N_{0}\left(u_{0}(x, t)\right) & \\
+\sum_{i=1}^{j-1} C_{i}\left[L\left(u_{j-1}(x, t)\right)\right. & \\
& \left.+N_{j-1}\left(u_{0}(x, t), \ldots, u_{j-1}(x, t)\right)\right] ; \\
& j=2,3, \ldots,
\end{aligned}
$$

where $N_{j}\left(u_{0}(x, t), \ldots, u_{j}(x, t)\right)$ is the coefficient of $p^{j}$ in the expansion of $N(\varphi(x, t ; p))$ about the embedding parameter $p$ and

$$
\begin{aligned}
& N\left(\varphi\left(x, t ; p, C_{1}, C_{2}, C_{3}, \ldots\right)\right) \\
& \quad=N_{0}\left(u_{0}(x, t)\right)+\sum_{j=1}^{\infty} N_{j}\left(u_{0}, u_{1}, \ldots, u_{j}\right) p^{j} .
\end{aligned}
$$

It is observed that the convergence of the series (24) depends upon the auxiliary constants $C_{1}, C_{2}, C_{3}, \ldots$.

The approximate solution of (18) can be written in the following form:

$$
\begin{aligned}
& \tilde{u}\left(x, t ; C_{1}, C_{2}, C_{3}, \ldots\right) \\
& =u_{0}(x, t)+\sum_{j=1}^{n-1} u_{j}\left(x, t, C_{1}, C_{2}, C_{3}, \ldots\right) .
\end{aligned}
$$

Substituting (28) in (18), we get the following expression for the residual

$$
\begin{aligned}
R_{n}\left(x, t ; C_{1}, C_{2}, C_{3}, \ldots\right)= & L\left(\widetilde{u}\left(x, t ; C_{1}, C_{2}, C_{3}, \ldots\right)\right) \\
& +N\left(\widetilde{u}\left(x, t ; C_{1}, C_{2}, C_{3}, \ldots\right)\right) \\
& +g(x, t) .
\end{aligned}
$$

If $R_{n}\left(x, t ; C_{1}, C_{2}, C_{3}, \ldots\right)=0$, then $\tilde{u}\left(x, t ; C_{1}, C_{2}, C_{3}, \ldots\right)$ is the exact solution. Generally such case does not arise for nonlinear problems. The $n$th order approximate solution given by (28) depends on the auxiliary constants $C_{1}, C_{2}, C_{3}, \ldots$ and these constants can be optimally determined by various methods such as weighted residual least square method, Galerkin method, and collocation method. Here we apply collocation method.

According to the collocation method the optimal values of the constants $C_{1}, C_{2}, C_{3}, \ldots$ can be obtained by solving the following system of equations:

$$
\begin{array}{r}
R_{n}\left(x_{i}, t_{j} ; C_{1}, C_{2}, C_{3}, \ldots, C_{k^{2}}\right)=0 \quad \text { for } i \\
=1,2, \ldots, k, \\
j=1,2, \ldots, k .
\end{array}
$$

The convergence of the $n$th approximate solution depends upon unknown constants $C_{1}, C_{2}, C_{3}, \ldots$. When the convergence control constants $C_{1}, C_{2}, C_{3}, \ldots$ are known by the above mentioned methods then the approximate solution of (18) is well determined.

\section{Application of Haar Wavelet to Fractional Order Burgers-Fisher Equation}

Consider the generalized one dimensional Burgers-Fisher equation [8] of fractional order

$$
\frac{\partial^{\alpha} u}{\partial t^{\alpha}}+\xi u^{\eta} \frac{\partial u}{\partial x}=\mu \frac{\partial^{2} u}{\partial x^{2}}+\beta u\left(1-u^{\eta}\right),
$$

where $\xi, \mu$ and $\beta$ are parameters and $0<\alpha \leq 1$, with the initial and boundary conditions

$$
u(x, 0)=\left[\frac{1}{2}+\frac{1}{2} \tanh \left(\frac{-\xi \eta}{2(\eta+1)} x\right)\right]^{1 / \eta},
$$

$u(0, t)$

$$
\begin{aligned}
=\left(\frac{1}{2}+\frac{1}{2} \tanh \right. & {\left[\frac{-\xi \eta}{2(\eta+1)}\right.} \\
& \left.\left.\times\left(-\left(\frac{\xi}{\eta+1}+\frac{\beta(\eta+1)}{\xi}\right) t\right)\right]\right)^{1 / \eta},
\end{aligned}
$$

$u(1, t)$

$$
\begin{aligned}
=\left(\frac{1}{2}+\frac{1}{2} \tanh [\right. & \frac{-\xi \eta}{2(\eta+1)} \\
& \left.\left.\times\left(1-\left(\frac{\xi}{\eta+1}+\frac{\beta(\eta+1)}{\xi}\right) t\right)\right]\right)^{1 / \eta} .
\end{aligned}
$$


When $\alpha=1$, the exact solution of (31) is given by [8]

$$
\begin{aligned}
u(x, t)=\left(\frac{1}{2}+\frac{1}{2} \tanh \right. & {\left[\frac{-\xi \eta}{2(\eta+1)}\right.} \\
& \left.\left.\times\left(x-\left(\frac{\xi}{\eta+1}+\frac{\beta(\eta+1)}{\xi}\right) t\right)\right]\right)^{1 / \eta} .
\end{aligned}
$$

Let us divide both space and time interval $[0,1]$ into $m$ equal subintervals; each of width $\Delta=1 / \mathrm{m}$. Here we have taken $\eta=$ $1, \mu=1$ and $\xi=\beta=0.01$. Therefore, (31) reduces to

$$
\frac{\partial^{\alpha} u}{\partial t^{\alpha}}-\frac{\partial^{2} u}{\partial x^{2}}+0.01\left(u \frac{\partial u}{\partial x}+u(u-1)\right)=0 .
$$

Haar wavelet solution of $u(x, t)$ is sought by assuming that $\partial^{2} u(x, t) / \partial x^{2}$ can be expanded in terms of Haar wavelets as

$$
\frac{\partial^{2} u(x, t)}{\partial x^{2}}=\sum_{i=1}^{m} \sum_{j=1}^{m} c_{i j} h_{i}(x) h_{j}(t) .
$$

Integrating (36) w.r.t. $x$ from 0 to $x$ we get

$$
\frac{\partial u(x, t)}{\partial x}-p(t)=\sum_{i=1}^{m} \sum_{j=1}^{m} c_{i j} Q h_{i}(x) h_{j}(t) .
$$

Again, integrating (37) w.r.t. $x$ from 0 to $x$ we get

$$
u(x, t)=\sum_{i=1}^{m} \sum_{j=1}^{m} c_{i j} Q^{2} h_{i}(x) h_{j}(t)+q(t)+x p(t) .
$$

Putting $x=0$, in (38) we get

$$
q(t)=u(0, t)
$$

Putting $x=1$, in (38) we get

$$
p(t)=u(1, t)-u(0, t)-\sum_{i=1}^{m} \sum_{j=1}^{m} c_{i j}\left[Q^{2} h_{i}(x)\right]_{x=1} h_{j}(t) .
$$

Again $q(t)+x p(t)$ can be approximated using Haar wavelet function as

$$
q(t)+x p(t)=\sum_{i=1}^{m} \sum_{j=1}^{m} r_{i j} h_{i}(x) h_{j}(t) .
$$

This implies

$$
\begin{aligned}
u(0, t)+x & {[u(1, t)-u(0, t)} \\
& \left.-\sum_{i=1}^{m} \sum_{j=1}^{m} c_{i j}\left[Q^{2} h_{i}(x)\right]_{x=1} h_{j}(t)\right] \\
= & \sum_{i=1}^{m} \sum_{j=1}^{m} r_{i j} h_{i}(x) h_{j}(t) .
\end{aligned}
$$

Substituting (41) in (38), we get

$$
\begin{aligned}
u(x, t)= & \sum_{i=1}^{m} \sum_{j=1}^{m} c_{i j} Q^{2} h_{i}(x) h_{j}(t) \\
& +\sum_{i=1}^{m} \sum_{j=1}^{m} r_{i j} h_{i}(x) h_{j}(t) .
\end{aligned}
$$

The nonlinear term presented in (35) can be approximated using Haar wavelet function as

$$
u \frac{\partial u}{\partial x}+u(u-1)=\sum_{i=1}^{m} \sum_{j=1}^{m} d_{i j} h_{i}(x) h_{j}(t) .
$$

Therefore from (37), (40), and (43) we have

$$
\begin{aligned}
\left(\sum_{i=1}^{m} \sum_{j=1}^{m} c_{i j} Q^{2} h_{i}(x) h_{j}(t)+\sum_{i=1}^{m} \sum_{j=1}^{m} r_{i j} h_{i}(x) h_{j}(t)\right) \\
\times\left[\sum_{i=1}^{m} \sum_{j=1}^{m} c_{i j} Q h_{i}(x) h_{j}(t)+u(1, t)-u(0, t)\right. \\
\left.\quad-\sum_{i=1}^{m} \sum_{j=1}^{m} c_{i j}\left[Q^{2} h_{i}(x)\right]_{x=1} h_{j}(t)\right] \\
+\left(\sum_{i=1}^{m} \sum_{j=1}^{m} c_{i j} Q^{2} h_{i}(x) h_{j}(t)+\sum_{i=1}^{m} \sum_{j=1}^{m} r_{i j} h_{i}(x) h_{j}(t)\right) \\
\quad \times\left(\sum_{i=1}^{m} \sum_{j=1}^{m} c_{i j} Q^{2} h_{i}(x) h_{j}(t)+\sum_{i=1}^{m} \sum_{j=1}^{m} r_{i j} h_{i}(x) h_{j}(t)-1\right) \\
=\sum_{i=1}^{m} \sum_{j=1}^{m} d_{i j} h_{i}(x) h_{j}(t) .
\end{aligned}
$$

Substituting (36) and (44) in (35) we will have

$$
\frac{\partial^{\alpha} u}{\partial t^{\alpha}}=\sum_{i=1}^{m} \sum_{j=1}^{m} c_{i j} h_{i}(x) h_{j}(t)-0.01 \sum_{i=1}^{m} \sum_{j=1}^{m} d_{i j} h_{i}(x) h_{j}(t) .
$$

Now applying $J^{\alpha}$ to both sides of (46) yields

$$
\begin{aligned}
u(x, t) & -u(x, 0) \\
= & J^{\alpha}\left(\sum_{i=1}^{m} \sum_{j=1}^{m} c_{i j} h_{i}(x) h_{j}(t)\right) \\
& -0.01 J^{\alpha}\left(\sum_{i=1}^{m} \sum_{j=1}^{m} d_{i j} h_{i}(x) h_{j}(t)\right) .
\end{aligned}
$$


Substituting (32) and (43) in (47) we get

$$
\begin{aligned}
\sum_{i=1}^{m} \sum_{j=1}^{m} c_{i j} Q^{2} h_{i}(x) h_{j}(t) & \\
& +\sum_{i=1}^{m} \sum_{j=1}^{m} r_{i j} h_{i}(x) h_{j}(t) \\
& -\left[\frac{1}{2}+\frac{1}{2} \tanh \left(\frac{-\xi \eta}{2(\eta+1)} x\right)\right] \\
= & \sum_{i=1}^{m} \sum_{j=1}^{m} c_{i j} h_{i}(x) Q_{t}^{\alpha} h_{j}(t) \\
& -0.01\left(\sum_{i=1}^{m} \sum_{j=1}^{m} d_{i j} h_{i}(x) Q_{t}^{\alpha} h_{j}(t)\right) .
\end{aligned}
$$

Now substituting the collocation points $x_{l}=(l-0.5) / m$ and $t_{k}=(k-0.5) / m$ for $l, k=1,2, \ldots, m$ in (42), (45), and (48), we have $3 m^{2}$ equations in $3 m^{2}$ unknowns in $c_{i j}$, $r_{i j}$ and $d_{i j}$. By solving this system of equations using mathematical software, the Haar wavelet coefficients $c_{i j}, r_{i j}$, and $d_{i j}$ can be obtained.

\section{Haar Wavelet Based Scheme for Generalized Fisher's Equation}

Consider the generalized Fisher's equation [9] of fractional order

$$
\frac{\partial^{\alpha} u}{\partial t^{\alpha}}=\frac{\partial^{2} u}{\partial x^{2}}+u\left(1-u^{6}\right)
$$

where $0<\alpha \leq 1$ and $0 \leq x \leq 1$ with the initial condition

$$
u(x, 0)=\frac{1}{\left(1+e^{(3 / 2) x}\right)^{1 / 3}} .
$$

When $\alpha=1$, the exact solution of (49) is given by [10]

$$
u(x, t)=\left(\frac{1}{2}+\frac{1}{2} \tanh \left[\frac{-3}{4}\left(x-\frac{5}{2} t\right)\right]\right)^{1 / 3} .
$$

Let us divide both space and time interval $[0,1]$ into $m$ equal subintervals; each of width $\Delta=1 / \mathrm{m}$.

The Haar wavelet solution of $u(x, t)$ is sought by assuming that $\partial^{2} u(x, t) / \partial x^{2}$ can be expanded in terms of Haar wavelets as

$$
\frac{\partial^{2} u(x, t)}{\partial x^{2}}=\sum_{i=1}^{m} \sum_{j=1}^{m} c_{i j} h_{i}(x) h_{j}(t) .
$$

Integrating (52) twice w.r.t. $x$ from 0 to $x$ we get

$$
u(x, t)=\sum_{i=1}^{m} \sum_{j=1}^{m} c_{i j} Q^{2} h_{i}(x) h_{j}(t)+q(t)+x p(t) .
$$

Putting $x=0$, in (53) we get

$$
q(t)=u(0, t)
$$

Putting $x=1$, in (53) we get

$$
p(t)=u(1, t)-u(0, t)-\sum_{i=1}^{m} \sum_{j=1}^{m} c_{i j}\left[Q^{2} h_{i}(x)\right]_{x=1} h_{j}(t) .
$$

Substituting (54) and (55) in (53) we have

$$
\begin{aligned}
u(x, t)= & \sum_{i=1}^{m} \sum_{j=1}^{m} c_{i j} Q^{2} h_{i}(x) h_{j}(t)+u(0, t) \\
& +x\left[u(1, t)-u(0, t)-\sum_{i=1}^{m} \sum_{j=1}^{m} c_{i j}\left[Q^{2} h_{i}(x)\right]_{x=1} h_{j}(t)\right] .
\end{aligned}
$$

The nonlinear term presented in (49) can be approximated using Haar wavelet function as

$$
u\left(1-u^{6}\right)=\sum_{i=1}^{m} \sum_{j=1}^{m} d_{i j} h_{i}(x) h_{j}(t)
$$

which implies

$$
\begin{aligned}
& \left(\sum_{i=1}^{m} \sum_{j=1}^{m} c_{i j} Q^{2} h_{i}(x) h_{j}(t)+u(0, t)\right. \\
& \left.+x\left[u(1, t)-u(0, t)-\sum_{i=1}^{m} \sum_{j=1}^{m} c_{i j}\left[Q^{2} h_{i}(x)\right]_{x=1} h_{j}(t)\right]\right) \\
& \times\left[1-\left(\sum_{i=1}^{m} \sum_{j=1}^{m} c_{i j} Q^{2} h_{i}(x) h_{j}(t)+u(0, t)\right.\right. \\
& +x[u(1, t)-u(0, t) \\
& \left.\left.\left.-\sum_{i=1}^{m} \sum_{j=1}^{m} c_{i j}\left[Q^{2} h_{i}(x)\right]_{x=1} h_{j}(t)\right]\right)^{6}\right] \\
& =\sum_{i=1}^{m} \sum_{j=1}^{m} d_{i j} h_{i}(x) h_{j}(t) .
\end{aligned}
$$

Substituting (52) and (57) in (49) we will have

$$
\frac{\partial^{\alpha} u}{\partial t^{\alpha}}=\sum_{i=1}^{m} \sum_{j=1}^{m} c_{i j} h_{i}(x) h_{j}(t)+\sum_{i=1}^{m} \sum_{j=1}^{m} d_{i j} h_{i}(x) h_{j}(t) .
$$

Now applying $J^{\alpha}$ to both sides of (59) yields

$$
\begin{aligned}
u(x, t)-u(x, 0)= & J^{\alpha}\left(\sum_{i=1}^{m} \sum_{j=1}^{m} c_{i j} h_{i}(x) h_{j}(t)\right) \\
& +J^{\alpha}\left(\sum_{i=1}^{m} \sum_{j=1}^{m} d_{i j} h_{i}(x) h_{j}(t)\right) .
\end{aligned}
$$


Substituting (50) and (56) in (60) we get

$$
\begin{aligned}
\sum_{i=1}^{m} \sum_{j=1}^{m} c_{i j} Q^{2} h_{i}(x) h_{j}(t)+u(0, t) & \\
& +x\left[u(1, t)-u(0, t)-\sum_{i=1}^{m} \sum_{j=1}^{m} c_{i j}\left[Q^{2} h_{i}(x)\right]_{x=1} h_{j}(t)\right] \\
& -\frac{1}{\left(1+e^{(3 / 2) x}\right)^{1 / 3}} \\
= & \sum_{i=1}^{m} \sum_{j=1}^{m} c_{i j} h_{i}(x) Q_{t}^{\alpha} h_{j}(t) \\
& +\sum_{i=1}^{m} \sum_{j=1}^{m} d_{i j} h_{i}(x) Q_{t}^{\alpha} h_{j}(t)
\end{aligned}
$$

Now substituting the collocation points $x_{l}=(l-0.5) / m$ and $t_{k}=(k-0.5) / m$ for $l, k=1,2, \ldots, m$ in (61) and (58), we have $2 m^{2}$ equations in $2 m^{2}$ unknowns in $c_{i j}$ and $d_{i j}$. By solving these system of equations using mathematical software, the Haar wavelet coefficients $c_{i j}$ and $d_{i j}$ can be obtained.

\section{Application of OHAM to Fractional Order Burgers-Fisher Equation}

Using optimal homotopy asymptotic method, the homotopy for (35) can be written as

$$
\begin{aligned}
&(1-p) \frac{\partial^{\alpha} \varphi(x, t ; p)}{\partial t^{\alpha}} \\
&=H(p)\left[\frac{\partial^{\alpha} \varphi(x, t ; p)}{\partial t^{\alpha}}-\frac{\partial^{2} \varphi(x, t ; p)}{\partial x^{2}}\right. \\
&+\varphi(x, t ; p) \frac{\partial \varphi(x, t ; p)}{\partial x} \\
&\quad-\varphi(x, t ; p)[1-\varphi(x, t ; p)]]
\end{aligned}
$$

Here,

$$
\begin{gathered}
\varphi(x, t ; p)=u_{0}(x, t)+\sum_{i=1}^{\infty} u_{i}(x, t) p^{i} \\
H(p)=p C_{1}+p^{2} C_{2}+p^{3} C_{3}+\cdots, \\
N(\varphi(x, t ; p))=N_{0}\left(u_{0}(x, t)\right)+\sum_{k=1}^{\infty} N_{k}\left(u_{0}, u_{1}, \ldots, u_{k}\right) p^{k} .
\end{gathered}
$$

Substituting (63) in (62) and equating the coefficients of like powers of $p$, we have the following system of partial differential equations.

Coefficients of $p^{0}$ are

$$
\frac{\partial^{\alpha} u_{0}(x, t)}{\partial t^{\alpha}}=0
$$

Coefficients of $p^{1}$ are

$$
\frac{\partial^{\alpha} u_{1}(x, t)}{\partial t^{\alpha}}-\frac{\partial^{\alpha} u_{0}(x, t)}{\partial t^{\alpha}}
$$

$$
\begin{aligned}
=C_{1}\left[\frac{\partial^{\alpha} u_{0}(x, t)}{\partial t^{\alpha}}-\frac{\partial^{2} u_{0}(x, t)}{\partial x^{2}}\right. \\
+0.01\left\{u_{0}(x, t) \frac{\partial u_{0}(x, t)}{\partial x}\right. \\
\left.\left.+\left(u_{0}(x, t)\right)^{2}-u_{0}(x, t)\right\}\right] .
\end{aligned}
$$

Coefficients of $p^{2}$ are

$$
\begin{gathered}
\frac{\partial^{\alpha} u_{2}(x, t)}{\partial t^{\alpha}}-\frac{\partial^{\alpha} u_{1}(x, t)}{\partial t^{\alpha}} \\
=C_{1}\left[\frac{\partial^{\alpha} u_{1}(x, t)}{\partial t^{\alpha}}-\frac{\partial^{2} u_{1}(x, t)}{\partial x^{2}}\right. \\
+0.01\left\{u_{0}(x, t) \frac{\partial u_{1}(x, t)}{\partial x}+2 u_{0}(x, t) u_{1}(x, t)\right. \\
\left.\left.+u_{1}(x, t) \frac{\partial u_{0}(x, t)}{\partial x}-u_{1}(x, t)\right\}\right] \\
+C_{2}\left[\frac{\partial^{\alpha} u_{0}(x, t)}{\partial t^{\alpha}}-\frac{\partial^{2} u_{0}(x, t)}{\partial x^{2}}\right. \\
+0.01\left\{u_{0}(x, t) \frac{\partial u_{0}(x, t)}{\partial x}\right. \\
\left.\left.+\left(u_{0}(x, t)\right)^{2}-u_{0}(x, t)\right\}\right]
\end{gathered}
$$

and so on.

For solving fractional order Burgers-Fisher equation using OHAM, we consider the initial condition (32), and solving (64) to (66), we obtain

$$
\begin{gathered}
u_{0}(x, t)=\left[\frac{1}{2}+\frac{1}{2} \tanh \left(\frac{-0.01}{4} x\right)\right], \\
u_{1}(x, t)=\frac{-0.00250625 C_{1} \operatorname{sech}^{2}(0.0025 x) t^{\alpha}}{\Gamma(1+\alpha)}, \\
u_{2}(x, t)=u_{1}(x, t) \\
+C_{1}\left[u_{1}(x, t)-\left(\left(C _ { 1 } \left(\operatorname{sech}^{4}(0.0025 x)\right.\right.\right.\right. \\
\left.\left.\quad \times \tanh ^{2}(0.0025 x)\right) t^{2 \alpha}\right) \\
\left.\times(\Gamma(1+2 \alpha))^{-1}\right) \\
+0.01\left\{\left(\left(C_{1} \operatorname{sech}^{2}(0.0025 x)\right.\right.\right. \\
\times\left(-1+\tanh ^{2}(0.0025 x)\right)
\end{gathered}
$$


TABLE 1: The absolute errors in the solution of fractional order Burgers-Fisher equation (35) using Haar wavelet method and three terms for second-order OHAM with convergence control parameters $C_{1}=0$ and $C_{2}=-0.99999$ at various points of $x$ and $t$ for $\alpha=1$.

\begin{tabular}{|c|c|c|c|c|c|c|c|c|}
\hline \multirow{2}{*}{$x$} & \multicolumn{4}{|c|}{$\left|u_{\text {Exact }}-u_{\text {Haar }}\right|$} & \multicolumn{4}{|c|}{$\left|u_{\text {Exact }}-u_{\text {OHAM }}\right|$} \\
\hline & $t=0.2$ & $t=0.4$ & $t=0.6$ & $t=0.8$ & $t=0.2$ & $t=0.4$ & $t=0.6$ & $t=0.8$ \\
\hline 0.1 & $5.4804 E-5$ & $2.3476 E-5$ & $7.8526 E-6$ & $3.9181 E-5$ & $4.229 E-11$ & $8.408 E-10$ & $3.403 E-9$ & $8.7368 E-9$ \\
\hline 0.2 & $2.3553 E-5$ & $7.7785 E-6$ & $3.9108 E-5$ & $7.0440 E-5$ & $8.333 E-11$ & $3.384 E-10$ & $2.273 E-9$ & $6.7268 E-9$ \\
\hline 0.3 & $7.0426 E-5$ & $3.9091 E-5$ & $7.7594 E-6$ & $2.3578 E-5$ & $2.089 E-10$ & $1.642 E-10$ & $1.142 E-9$ & $4.7168 E-9$ \\
\hline 0.4 & $3.9169 E-5$ & $7.8222 E-6$ & $2.35157 E-5$ & $5.4870 E-5$ & $3.346 E-10$ & $6.667 E-10$ & $1.13 E-11$ & $2.7068 E-9$ \\
\hline 0.5 & $7.9054 E-6$ & $2.3463 E-5$ & $5.48121 E-5$ & $8.6199 E-6$ & $4.602 E-10$ & $1.1692 E-9$ & $1.119 E-9$ & $6.968 E-10$ \\
\hline 0.6 & $5.4768 E-5$ & $2.3384 E-5$ & $7.97308 E-6$ & $3.9384 E-5$ & $5.858 E-10$ & $1.6717 E-9$ & $2.249 E-9$ & $1.3132 E-9$ \\
\hline 0.7 & $2.3489 E-5$ & $7.9370 E-6$ & $3.93167 E-5$ & $7.0791 E-5$ & $7.115 E-10$ & $2.1742 E-9$ & $3.381 E-9$ & $3.3232 E-9$ \\
\hline 0.8 & $7.0337 E-5$ & $3.8884 E-5$ & $7.4894 E-6$ & $2.4026 E-5$ & $8.371 E-10$ & $2.6767 E-9$ & $4.511 E-9$ & $5.3332 E-9$ \\
\hline 0.9 & $3.9031 E-5$ & $7.5074 E-6$ & $2.39232 E-5$ & $5.5543 E-5$ & $9.627 E-10$ & $3.1792 E-9$ & $5.642 E-9$ & $7.3432 E-9$ \\
\hline 1.0 & $8.5852 E-5$ & $5.4286 E-5$ & $2.28326 E-5$ & $8.8514 E-6$ & $1.0883 E-9$ & $3.6817 E-9$ & $6.772 E-9$ & $9.3532 E-9$ \\
\hline
\end{tabular}

TABLE 2: The absolute errors in the solution of generalized Fisher's equation (49) using Haar wavelet method and five terms for fourth-order OHAM with convergence control parameters $C_{1}=-0.637012, C_{2}=-0.151156, C_{3}=0.023432$, and $C_{4}=-0.0012788$ at various points of $x$ and $t$ for $\alpha=1$.

\begin{tabular}{|c|c|c|c|c|c|c|c|c|}
\hline \multirow{2}{*}{$x$} & \multicolumn{4}{|c|}{$\left|u_{\text {Exact }}-u_{\text {Haar }}\right|$} & \multicolumn{4}{|c|}{$\left|u_{\text {Exact }}-u_{\text {OHAM }}\right|$} \\
\hline & $t=0.2$ & $t=0.4$ & $t=0.6$ & $t=0.8$ & $t=0.2$ & $t=0.4$ & $t=0.6$ & $t=0.8$ \\
\hline 0.1 & 0.0051104 & 0.0054849 & 0.0042629 & 0.0027556 & $6.67439 E-5$ & 0.0040747 & 0.023752 & 0.0740709 \\
\hline 0.2 & 0.0096957 & 0.0106181 & 0.0085377 & 0.0058225 & $1.99503 E-4$ & 0.0023417 & 0.019345 & 0.0678136 \\
\hline 0.3 & 0.013553 & 0.0151142 & 0.0125286 & 0.0089272 & $4.65337 E-4$ & $4.3112 E-4$ & 0.013810 & 0.0581267 \\
\hline 0.4 & 0.0163976 & 0.018593 & 0.0158324 & 0.0116856 & $7.18406 E-4$ & 0.0015578 & 0.007399 & 0.0453319 \\
\hline 0.5 & 0.0179265 & 0.0206477 & 0.0180051 & 0.0136684 & $9.47663 E-4$ & 0.0035178 & $4.521 E-4$ & 0.030035 \\
\hline 0.6 & 0.017924 & 0.0209528 & 0.0186691 & 0.0145062 & 0.0011444 & 0.0053436 & 0.006645 & 0.013062 \\
\hline 0.7 & 0.0163414 & 0.01935 & 0.0175984 & 0.013973 & 0.0013029 & 0.0069445 & 0.013494 & 0.0046328 \\
\hline 0.8 & 0.0133823 & 0.015939 & 0.0148102 & 0.0120755 & 0.0014207 & 0.0082540 & 0.019736 & 0.0220718 \\
\hline 0.9 & 0.0095361 & 0.0111158 & 0.0106057 & 0.0090918 & 0.0014982 & 0.009231 & 0.025078 & 0.0383517 \\
\hline 1.0 & 0.0055837 & 0.0055837 & 0.0055837 & 0.0055837 & 0.0015382 & 0.0098632 & 0.029315 & 0.0527218 \\
\hline
\end{tabular}

$$
\begin{gathered}
\left.\times \tanh (0.0025 x) t^{2 \alpha}\right) \\
\left.\times(\Gamma(1+2 \alpha))^{-1}\right) \\
+\left(\left(C_{1} \operatorname{sech}^{4}(0.0025 x) t^{2 \alpha}\right)\right. \\
\left.\times(\Gamma(1+2 \alpha))^{-1}\right) \\
+\left(\left(0.00250625 C_{1} \operatorname{sech}^{2}(0.0025 x)\right.\right. \\
\left.\times\left(-1+\tanh ^{2}(0.0025 x)\right) t^{2 \alpha}\right) \\
\left.\times(\Gamma(1+2 \alpha))^{-1}\right) \\
-( \\
\times\left(0.00250625 C_{1}\right. \\
\left.+\operatorname{sech}_{2}^{2}(0.0025 x) t^{2 \alpha}\right) \\
\left.\left.\left.\times(\Gamma(1+2 \alpha))^{-1}\right)\right\}\right] \\
-\frac{\partial^{2} u_{0}(x, t)}{\partial x^{2}}
\end{gathered}
$$

$$
\begin{aligned}
& +0.01\left\{u_{0}(x, t) \frac{\partial u_{0}(x, t)}{\partial x}\right. \\
& \left.\left.+\left(u_{0}(x, t)\right)^{2}-u_{0}(x, t)\right\}\right] \\
& \times \frac{t^{\alpha}}{\Gamma(1+\alpha)} .
\end{aligned}
$$

Using (67) and consequently substituting in (28), the second order approximate solution is obtained as follows:

$$
\begin{aligned}
u(x, t)= & {\left[\frac{1}{2}+\frac{1}{2} \tanh \left(\frac{-0.01}{4} x\right)\right] } \\
& -\frac{0.00250625 C_{1} \operatorname{sech}^{2}(0.0025 x) t^{\alpha}}{\Gamma(1+\alpha)} \\
& +u_{1}(x, t)+C_{1}\left[u_{1}(x, t)\right. \\
-\left(\left(C _ { 1 } \left(\operatorname{sech}^{4}(0.0025 x)\right.\right.\right. & -2 \operatorname{sech}^{2}(0.0025 x)
\end{aligned}
$$


TABLE 3: The approximate solutions of fractional order Burgers-Fisher equation (35) using Haar wavelet method and three terms for secondorder OHAM with convergence control parameters $C_{1}=-0.000104528$ and $C_{2}=-0.99979$ at various points of $x$ and $t$ for $\alpha=0.75$.

\begin{tabular}{ccccccccc}
\hline \multirow{2}{*}{$t=0.2$} & \multicolumn{2}{c}{$t=0.4$} & \multicolumn{2}{c}{$t=0.6$} & \multicolumn{2}{c}{$t=0.8$} \\
& $u_{\text {Haar }}$ & $u_{\text {OHAM }}$ & $u_{\text {Haar }}$ & $u_{\text {OHAM }}$ & $u_{\text {Haar }}$ & $u_{\text {OHAM }}$ & $u_{\text {Haar }}$ \\
\hline 0.1 & 0.50043 & 0.500691 & 0.5009 & 0.501247 & 0.501369 & 0.501734 & 0.501839 \\
0.2 & 0.500271 & 0.500566 & 0.50074 & 0.501122 & 0.501207 & 0.501609 & 0.501674 \\
0.3 & 0.500187 & 0.500441 & 0.500656 & 0.500997 & 0.501119 & 0.501484 & 0.501584 \\
0.4 & 0.500028 & 0.500316 & 0.500495 & 0.500872 & 0.500944 & 0.501359 & 0.5014 & 0.502182 \\
0.5 & 0.499873 & 0.500191 & 0.500335 & 0.500747 & 0.500758 & 0.501234 & 0.501197 \\
0.6 & 0.499789 & 0.500066 & 0.500248 & 0.500622 & 0.500653 & 0.501109 & 0.50108 \\
0.7 & 0.499641 & 0.499941 & 0.500092 & 0.500497 & 0.500446 & 0.500984 & 0.500839 \\
0.8 & 0.499559 & 0.499816 & 0.500005 & 0.500372 & 0.500326 & 0.500859 & 0.501807 \\
0.9 & 0.499423 & 0.499691 & 0.499855 & 0.500247 & 0.500092 & 0.500734 & 0.501682 \\
1.0 & 0.499345 & 0.499566 & 0.499767 & 0.500122 & 0.499953 & 0.500609 & 0.501432 \\
\hline
\end{tabular}

TABLE 4: The approximate solutions of fractional order Burgers-Fisher equation (35) using Haar wavelet method and three terms for secondorder OHAM with convergence control parameters $C_{1}=0.000163239$ and $C_{2}=-1.00032796$ at various points of $x$ and $t$ for $\alpha=0.5$.

\begin{tabular}{ccccccccc}
\hline$x$ & \multicolumn{2}{c}{$t=0.2$} & \multicolumn{2}{c}{$t=0.4$} & \multicolumn{2}{c}{$t=0.6$} & \multicolumn{2}{c}{$t=0.8$} \\
& $u_{\text {Haar }}$ & $u_{\text {OHAM }}$ & $u_{\text {Haar }}$ & $u_{\text {OHAM }}$ & $u_{\text {Haar }}$ & $u_{\text {OHAM }}$ & $u_{\text {Haar }}$ \\
\hline 0.1 & 0.500429 & 0.50114 & 0.500898 & 0.501664 & 0.501368 & 0.502066 & 0.501837 & 0.502404 \\
0.2 & 0.500273 & 0.501015 & 0.500736 & 0.501539 & 0.501201 & 0.501941 & 0.501666 & 0.502279 \\
0.3 & 0.50019 & 0.50089 & 0.500646 & 0.501414 & 0.501105 & 0.501816 & 0.501566 & 0.502154 \\
0.4 & 0.500056 & 0.500765 & 0.500484 & 0.501289 & 0.500921 & 0.501691 & 0.501363 & 0.502029 \\
0.5 & 0.499953 & 0.50064 & 0.500329 & 0.501164 & 0.500725 & 0.501566 & 0.501132 & 0.501904 \\
0.6 & 0.499898 & 0.500515 & 0.500238 & 0.501039 & 0.500605 & 0.501441 & 0.500986 & 0.501779 \\
0.7 & 0.49986 & 0.50039 & 0.500098 & 0.500914 & 0.500386 & 0.501316 & 0.500698 & 0.501654 \\
0.8 & 0.499838 & 0.500265 & 0.50001 & 0.500789 & 0.500246 & 0.501191 & 0.500513 & 0.501529 \\
0.9 & 0.499889 & 0.50014 & 0.499894 & 0.500664 & 0.499999 & 0.501066 & 0.500153 & 0.501404 \\
1.0 & 0.499912 & 0.500015 & 0.499814 & 0.500539 & 0.499838 & 0.500941 & 0.499922 & 0.501279 \\
\hline
\end{tabular}

$$
\begin{aligned}
& \left.\left.\times \tanh ^{2}(0.0025 x)\right) t^{2 \alpha}\right) \\
\left.\times(\Gamma(1+2 \alpha))^{-1}\right) & \\
+0.01\{( & \left(C_{1} \operatorname{sech}^{2}(0.0025 x)\right. \\
& \times(-1+\tanh (0.0025 x)) \\
& \left.\times \tanh (0.0025 x) t^{2 \alpha}\right) \\
\times & \left.(\Gamma(1+2 \alpha))^{-1}\right) \\
+ & \frac{C_{1} \operatorname{sech}^{4}(0.0025 x) t^{2 \alpha}}{\Gamma(1+2 \alpha)} \\
+ & \left(\left(0.00250625 C_{1}\right.\right. \\
& \times \operatorname{sech}^{2}(0.0025 x) \\
& \times(-1+\tanh (0.0025 x)) \\
& \left.\times t^{2 \alpha}\right)
\end{aligned}
$$

$$
\begin{aligned}
& \left.\times(\Gamma(1+2 \alpha))^{-1}\right) \\
& -\left(\left(0.00250625 C_{1}\right.\right. \\
& \left.\quad \times \operatorname{sech}^{2}(0.0025 x) t^{2 \alpha}\right) \\
& \left.\left.\left.\quad \times(\Gamma(1+2 \alpha))^{-1}\right)\right\}\right]
\end{aligned}
$$$$
+C_{2}\left[-\frac{\partial^{2} u_{0}(x, t)}{\partial x^{2}}\right.
$$$$
+0.01\left\{u_{0}(x, t) \frac{\partial u_{0}(x, t)}{\partial x}\right.
$$$$
\left.\left.+\left(u_{0}(x, t)\right)^{2}-u_{0}(x, t)\right\}\right]
$$$$
\times \frac{t^{\alpha}}{\Gamma(1+\alpha)}
$$

The optimal values of the convergence control constants $C_{1}$ and $C_{2}$ can be obtained using collocation method from (30). 
TABLE 5: The approximate solutions of fractional order Burgers-Fisher equation (35) using Haar wavelet method and three terms for secondorder OHAM with convergence control parameters $C_{1}=-0.00019986$ and $C_{2}=-0.999602$ at various points of $x$ and $t$ for $\alpha=0.25$.

\begin{tabular}{ccccccccc}
\hline \multirow{2}{*}{$t=0.2$} & \multicolumn{2}{c}{$t=0.4$} & \multicolumn{2}{c}{$t=0.6$} & \multicolumn{2}{c}{$t=0.8$} \\
& $u_{\text {Haar }}$ & $u_{\text {OHAM }}$ & $u_{\text {Haar }}$ & $u_{\text {OHAM }}$ & $u_{\text {Haar }}$ & $u_{\text {OHAM }}$ & $u_{\text {Haar }}$ \\
\hline 0.1 & 0.500427 & 0.501724 & 0.500897 & 0.502074 & 0.501366 & 0.502309 & 0.501836 \\
0.2 & 0.50027 & 0.501599 & 0.500733 & 0.501949 & 0.501196 & 0.502184 & 0.50166 \\
0.3 & 0.500184 & 0.501474 & 0.500639 & 0.501824 & 0.501095 & 0.502059 & 0.501552 & 0.50249 \\
0.4 & 0.500056 & 0.501349 & 0.50048 & 0.501699 & 0.500909 & 0.501934 & 0.50134 & 0.502115 \\
0.5 & 0.49997 & 0.501224 & 0.500339 & 0.501574 & 0.500717 & 0.501809 & 0.501099 \\
0.6 & 0.49992 & 0.501099 & 0.500248 & 0.501449 & 0.500591 & 0.501684 & 0.500937 \\
0.7 & 0.499923 & 0.500974 & 0.500142 & 0.501324 & 0.500385 & 0.501559 & 0.500637 \\
0.8 & 0.499916 & 0.500849 & 0.500064 & 0.501199 & 0.500243 & 0.501434 & 0.500432 \\
0.9 & 0.500043 & 0.500724 & 0.500011 & 0.501074 & 0.500027 & 0.501309 & 0.501865 \\
1.0 & 0.500097 & 0.500599 & 0.499954 & 0.500949 & 0.499869 & 0.501184 & 0.500059 & 0.501615 \\
\hline
\end{tabular}

TABLE 6: The approximate solutions of generalized Fisher equation (49) using Haar wavelet method and five terms for fourth-order OHAM with convergence control parameters $C_{1}=-0.649458, C_{2}=0.053658, C_{3}=-0.1822726, C 4=0.0894301$ at various points of $x$ and $t$ for $\alpha=0.75$.

\begin{tabular}{|c|c|c|c|c|c|c|c|c|}
\hline \multirow{2}{*}{$x$} & \multicolumn{2}{|c|}{$t=0.2$} & \multicolumn{2}{|c|}{$t=0.4$} & \multicolumn{2}{|c|}{$t=0.6$} & \multicolumn{2}{|c|}{$t=0.8$} \\
\hline & $u_{\text {Haar }}$ & $u_{\text {OHAM }}$ & $u_{\text {Haar }}$ & $u_{\text {OHAM }}$ & $u_{\text {Haar }}$ & $u_{\text {OHAM }}$ & $u_{\text {Haar }}$ & $u_{\mathrm{OHAM}}$ \\
\hline 0.1 & 0.859185 & 0.899389 & 0.920547 & 0.931947 & 0.957952 & 0.927394 & 0.978665 & 0.902524 \\
\hline 0.2 & 0.838644 & 0.888856 & 0.905313 & 0.930017 & 0.948042 & 0.930519 & 0.972681 & 0.905646 \\
\hline 0.3 & 0.817612 & 0.877253 & 0.889588 & 0.927859 & 0.93764 & 0.934718 & 0.966206 & 0.91116 \\
\hline 0.4 & 0.796431 & 0.864492 & 0.873714 & 0.92523 & 0.927088 & 0.939825 & 0.959581 & 0.919341 \\
\hline 0.5 & 0.775484 & 0.850503 & 0.858074 & 0.921857 & 0.916772 & 0.945568 & 0.953191 & 0.930278 \\
\hline 0.6 & 0.755093 & 0.835244 & 0.84299 & 0.917456 & 0.90701 & 0.951569 & 0.947357 & 0.943809 \\
\hline 0.7 & 0.735424 & 0.818704 & 0.828628 & 0.911753 & 0.897971 & 0.957356 & 0.942244 & 0.959482 \\
\hline 0.8 & 0.716399 & 0.800908 & 0.81491 & 0.904497 & 0.889575 & 0.962388 & 0.937776 & 0.976567 \\
\hline 0.9 & 0.697656 & 0.781918 & 0.801474 & 0.895481 & 0.881463 & 0.966095 & 0.933591 & 0.994112 \\
\hline 1.0 & 0.678526 & 0.761827 & 0.787651 & 0.884559 & 0.872963 & 0.967927 & 0.929017 & 1.01104 \\
\hline
\end{tabular}

\section{Application of OHAM to Generalized Fisher's Equation}

Using optimal homotopy asymptotic method, the homotopy for (49) can be written as

$$
\begin{aligned}
&(1-p) \frac{\partial^{\alpha} \varphi(x, t ; p)}{\partial t^{\alpha}} \\
&=H(p)\left[\frac{\partial^{\alpha} \varphi(x, t ; p)}{\partial t^{\alpha}}-\frac{\partial^{2} \varphi(x, t ; p)}{\partial x^{2}}\right. \\
&\left.-\varphi(x, t ; p)\left[1-(\varphi(x, t ; p))^{6}\right]\right] .
\end{aligned}
$$

Here,

$$
\begin{gathered}
\varphi(x, t ; p)=u_{0}(x, t)+\sum_{i=1}^{\infty} u_{i}(x, t) p^{i}, \\
H(p)=p C_{1}+p^{2} C_{2}+p^{3} C_{3}+\cdots, \\
N(\varphi(x, t ; p))=N_{0}\left(u_{0}(x, t)\right)+\sum_{k=1}^{\infty} N_{k}\left(u_{0}, u_{1}, \ldots, u_{k}\right) p^{k} .
\end{gathered}
$$

Substituting (70) in (69) and equating the coefficients of like powers of $p$, we have the following system of partial differential equations.

Coefficients of $p^{0}$ are

$$
\frac{\partial^{\alpha} u_{0}(x, t)}{\partial t^{\alpha}}=0 .
$$

Coefficients of $p^{1}$ are

$$
\begin{aligned}
& \frac{\partial^{\alpha} u_{1}(x, t)}{\partial t^{\alpha}}-\frac{\partial^{\alpha} u_{0}(x, t)}{\partial t^{\alpha}} \\
& \quad=C_{1}\left[\frac{\partial^{\alpha} u_{0}(x, t)}{\partial t^{\alpha}}-\frac{\partial^{2} u_{0}(x, t)}{\partial x^{2}}+\left(u_{0}(x, t)\right)^{7}-u_{0}(x, t)\right] .
\end{aligned}
$$

Coefficients of $p^{2}$ are

$$
\begin{aligned}
& \frac{\partial^{\alpha} u_{2}(x, t)}{\partial t^{\alpha}}-\frac{\partial^{\alpha} u_{1}(x, t)}{\partial t^{\alpha}} \\
& =C_{1}\left[\frac{\partial^{\alpha} u_{1}(x, t)}{\partial t^{\alpha}}-\frac{\partial^{2} u_{1}(x, t)}{\partial x^{2}}\right.
\end{aligned}
$$


TABLE 7: The approximate solutions of generalized Fisher equation (49) using Haar wavelet method and five terms for fourth-order OHAM with convergence control parameters $C_{1}=-0.5059152, C_{2}=-0.0211535, C_{3}=-0.05081612$, and $C_{4}=0.0574318$ at various points of $x$ and $t$ for $\alpha=0.5$.

\begin{tabular}{|c|c|c|c|c|c|c|c|c|}
\hline \multirow{2}{*}{$x$} & \multicolumn{2}{|c|}{$t=0.2$} & \multicolumn{2}{|c|}{$t=0.4$} & \multicolumn{2}{|c|}{$t=0.6$} & \multicolumn{2}{|c|}{$t=0.8$} \\
\hline & $u_{\text {Haar }}$ & $u_{\text {OHAM }}$ & $u_{\text {Haar }}$ & $u_{\mathrm{OHAM}}$ & $u_{\text {Haar }}$ & $u_{\text {OHAM }}$ & $u_{\text {Haar }}$ & $u_{\text {OHAM }}$ \\
\hline 0.1 & 0.859121 & 0.927731 & 0.920482 & 0.943924 & 0.957888 & 0.943779 & 0.9786 & 0.939948 \\
\hline 0.2 & 0.838402 & 0.921616 & 0.905071 & 0.943123 & 0.947799 & 0.94491 & 0.972438 & 0.940694 \\
\hline 0.3 & 0.817123 & 0.914826 & 0.889099 & 0.942438 & 0.937151 & 0.946615 & 0.965717 & 0.941956 \\
\hline 0.4 & 0.795694 & 0.907206 & 0.872977 & 0.941713 & 0.926351 & 0.948908 & 0.958844 & 0.944068 \\
\hline 0.5 & 0.774576 & 0.898605 & 0.857166 & 0.940787 & 0.915864 & 0.951841 & 0.952284 & 0.947508 \\
\hline 0.6 & 0.754149 & 0.888882 & 0.842046 & 0.93948 & 0.906066 & 0.955446 & 0.946413 & 0.952761 \\
\hline 0.7 & 0.734584 & 0.877915 & 0.827789 & 0.937586 & 0.897131 & 0.959662 & 0.941405 & 0.96016 \\
\hline 0.8 & 0.715744 & 0.865607 & 0.814255 & 0.934862 & 0.888921 & 0.964283 & 0.937121 & 0.969739 \\
\hline 0.9 & 0.697156 & 0.851889 & 0.800975 & 0.931031 & 0.880963 & 0.968937 & 0.933091 & 0.98117 \\
\hline 1.0 & 0.678052 & 0.83673 & 0.787178 & 0.925803 & 0.872489 & 0.973109 & 0.928543 & 0.993777 \\
\hline
\end{tabular}

TABLE 8: The approximate solutions of generalized Fisher equation (49) using Haar wavelet method and five terms for fourth-order OHAM with convergence control parameters $C_{1}=-0.33833012, C_{2}=-0.04303056, C_{3}=0.1230816$, and $C_{4}=-0.0545852$ at various points of $x$ and $t$ for $\alpha=0.25$.

\begin{tabular}{|c|c|c|c|c|c|c|c|c|}
\hline \multirow{2}{*}{$x$} & \multicolumn{2}{|c|}{$t=0.2$} & \multicolumn{2}{|c|}{$t=0.4$} & \multicolumn{2}{|c|}{$t=0.6$} & \multicolumn{2}{|c|}{$t=0.8$} \\
\hline & $u_{\text {Haar }}$ & $u_{\mathrm{OHAM}}$ & $u_{\text {Haar }}$ & $u_{\mathrm{OHAM}}$ & $u_{\text {Haar }}$ & $u_{\mathrm{OHAM}}$ & $u_{\text {Haar }}$ & $u_{\mathrm{OHAM}}$ \\
\hline 0.1 & 0.859016 & 0.949105 & 0.920377 & 0.959635 & 0.957783 & 0.964066 & 0.978495 & 0.966453 \\
\hline 0.2 & 0.837987 & 0.943681 & 0.904655 & 0.955839 & 0.947384 & 0.960802 & 0.972023 & 0.963262 \\
\hline 0.3 & 0.816234 & 0.937961 & 0.88821 & 0.9522 & 0.936261 & 0.957955 & 0.964827 & 0.960634 \\
\hline 0.4 & 0.79426 & 0.93184 & 0.871543 & 0.948657 & 0.924917 & 0.95553 & 0.95741 & 0.958645 \\
\hline 0.5 & 0.772676 & 0.925203 & 0.855266 & 0.945143 & 0.913963 & 0.953545 & 0.950383 & 0.957415 \\
\hline 0.6 & 0.752001 & 0.917922 & 0.839899 & 0.941569 & 0.903919 & 0.951996 & 0.944265 & 0.957051 \\
\hline 0.7 & 0.732474 & 0.909857 & 0.825678 & 0.937799 & 0.895021 & 0.950809 & 0.939295 & 0.957571 \\
\hline 0.8 & 0.713871 & 0.900851 & 0.812383 & 0.933634 & 0.887048 & 0.949804 & 0.935249 & 0.958845 \\
\hline 0.9 & 0.695534 & 0.890742 & 0.799352 & 0.928815 & 0.87934 & 0.948678 & 0.931468 & 0.96056 \\
\hline 1.0 & 0.676507 & 0.87937 & 0.785633 & 0.923035 & 0.870944 & 0.947027 & 0.926998 & 0.96224 \\
\hline
\end{tabular}

$$
\begin{gathered}
\left.+7\left(u_{0}(x, t)\right)^{6} u_{1}(x, t)-u_{1}(x, t)\right] \\
+C_{1}\left[\frac{\partial^{\alpha} u_{0}(x, t)}{\partial t^{\alpha}}-\frac{\partial^{2} u_{0}(x, t)}{\partial x^{2}}\right. \\
\left.+\left(u_{0}(x, t)\right)^{7}-u_{0}(x, t)\right]
\end{gathered}
$$

and so on.

We consider the initial condition (50), and solving above equations, we obtain

$$
\begin{gathered}
u_{0}(x, t)=\frac{1}{\left(1+e^{(3 / 2) x}\right)^{1 / 3}}, \\
u_{1}(x, t)=\frac{-5 C_{1} e^{3 x / 2} t^{\alpha}}{4\left(1+e^{3 x / 2}\right)^{4 / 3} \Gamma(1+\alpha)}
\end{gathered}
$$

and so on.
From (28), the fourth order approximate solution can be obtained by using the formula

$$
u(x, t)=u_{0}(x, t)+u_{1}(x, t)+u_{2}(x, t)+u_{3}(x, t)+u_{4}(x, t) .
$$

The optimal values of the convergence control constants $C_{1}, C_{2}, C_{3}$, and $C_{4}$ can be obtained using collocation method from (30).

\section{Numerical Results and Discussion}

Tables 1 and 2, respectively, show the comparison of the absolute errors of fractional order Burgers-Fisher equation (35) and generalized Fisher's equation (49) obtained by using Haar wavelet method and OHAM at different values of $x$ and $t$. In Tables 1 to $8, m$ has been taken as 16 .

Tables 3, 4, and 5 exhibit the comparison of approximate solutions obtained by Haar wavelet method and OHAM for fractional order Burgers-Fisher equation (35). Similarly, Tables 6,7 , and 8 present the comparison of approximate solutions obtained by Haar wavelet method and OHAM for fractional order generalized Fisher's equation (49). The 


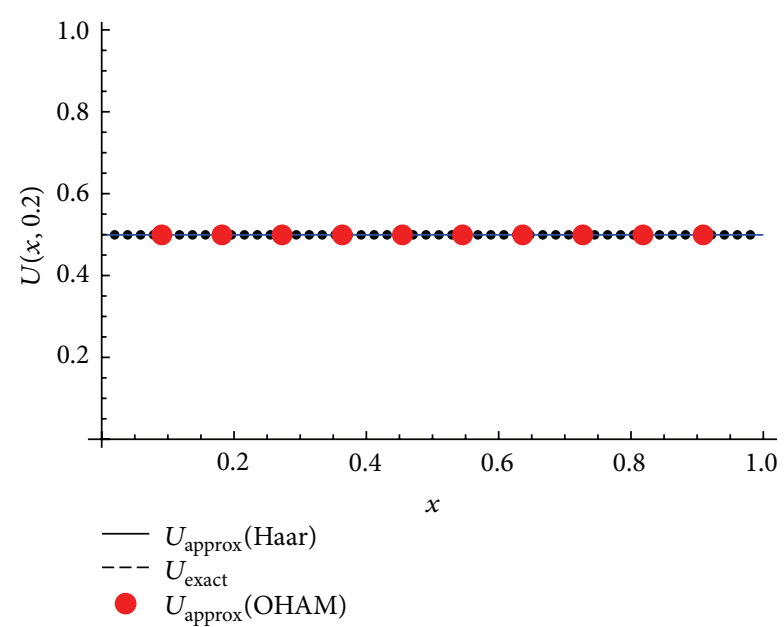

FIgURE 1: Comparison of Haar wavelet solutions and OHAM solutions with the exact solution of Burgers-Fisher equation when $t=0.2$.

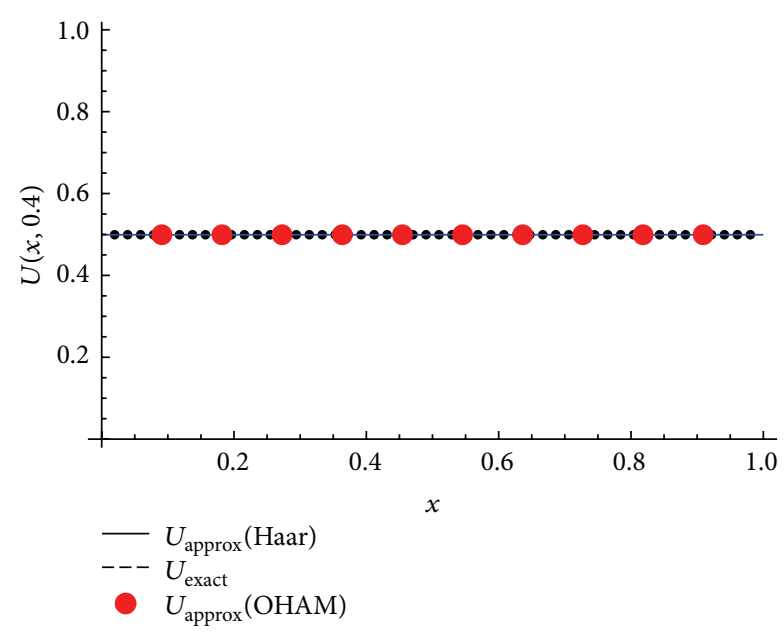

FIgURE 2: Comparison of Haar wavelet solutions and OHAM solutions with the exact solution of Burgers-Fisher equation when $t=0.4$.

obtained results in these Tables 1 to 8 demonstrate that these methods are well suited for solving fractional BurgersFisher and fractional generalized Fisher's equation. Both of the methods are quite efficient and effective.

Tables 9, 10, and 11 show the values of the Haar wavelet coefficients $c_{i j}, r_{i j}$ and $d_{i j}$ for fractional order Burgers-Fisher equation obtained from (42), (45), and (48).

In case of Burgers-Fisher equation, Figures 1 and 2 cite the comparison graphically between the numerical solutions obtained by Haar wavelet method, optimal homotopy asymptotic method (OHAM), and exact solutions for different values of $t$ and $x$. Similarly, in case of generalized Fisher's equation, Figures 3 and 4 present the comparison graphically between the numerical results obtained by Haar wavelet method, OHAM, and exact solutions for different values of $t$ and $x$.

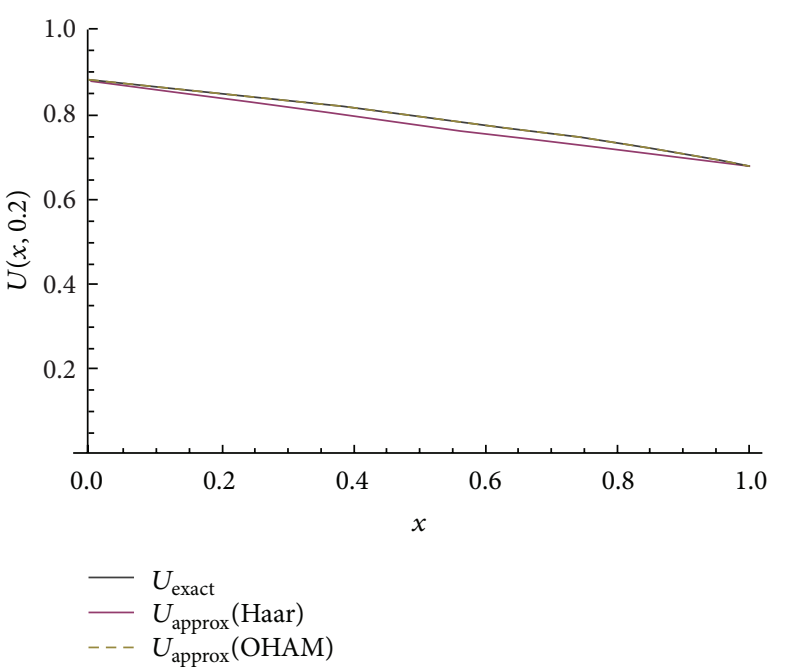

FIGURE 3: Comparison of Haar wavelet solutions and OHAM solutions with the exact solution of generalized Fisher's equation when $t=0.2$.

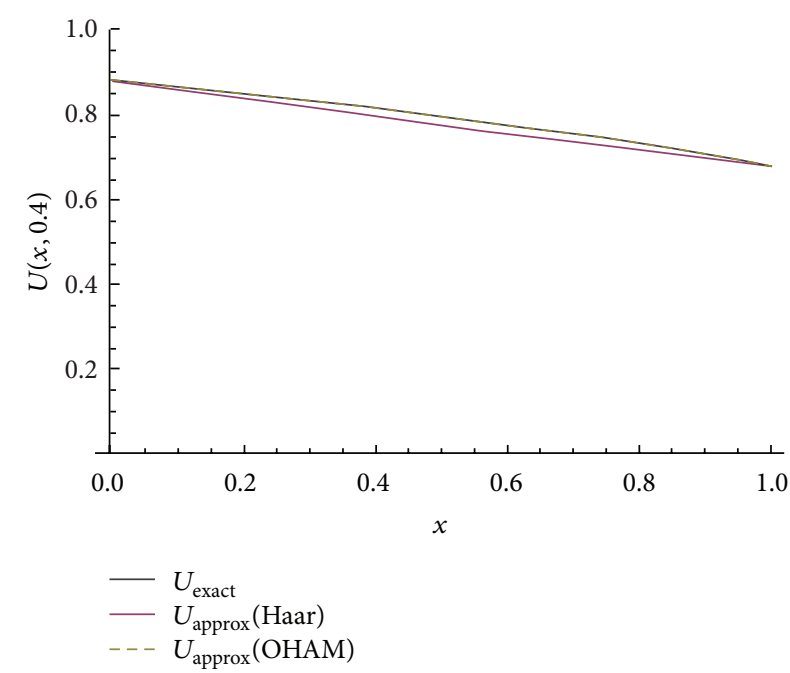

FIGURE 4: Comparison of Haar wavelet solutions and OHAM solutions with the exact solution of generalized Fisher's equation when $t=0.4$.

\section{Conclusion}

In this paper, the fractional order Burgers-Fisher and generalized Fisher's equations have been solved by using Haar wavelet method. The obtained results are then compared with exact solutions as well as optimal homotopy asymptotic method (OHAM). These results have been cited in the tables in order to justify the accuracy and efficiency of the proposed schemes. The Haar wavelet technique provides quite satisfactory results for the fractional order Burgers-Fisher (35) and generalized Fisher equations (49). The main advantages of this Haar wavelet method is that they transfer the whole scheme into a system of algebraic equations for which the computation is easy and simple. OHAM allows fine tuning of convergence region and rate of convergence by suitably identifying convergence control parameters $C_{1}, C_{2}, C_{3}, \ldots$. 


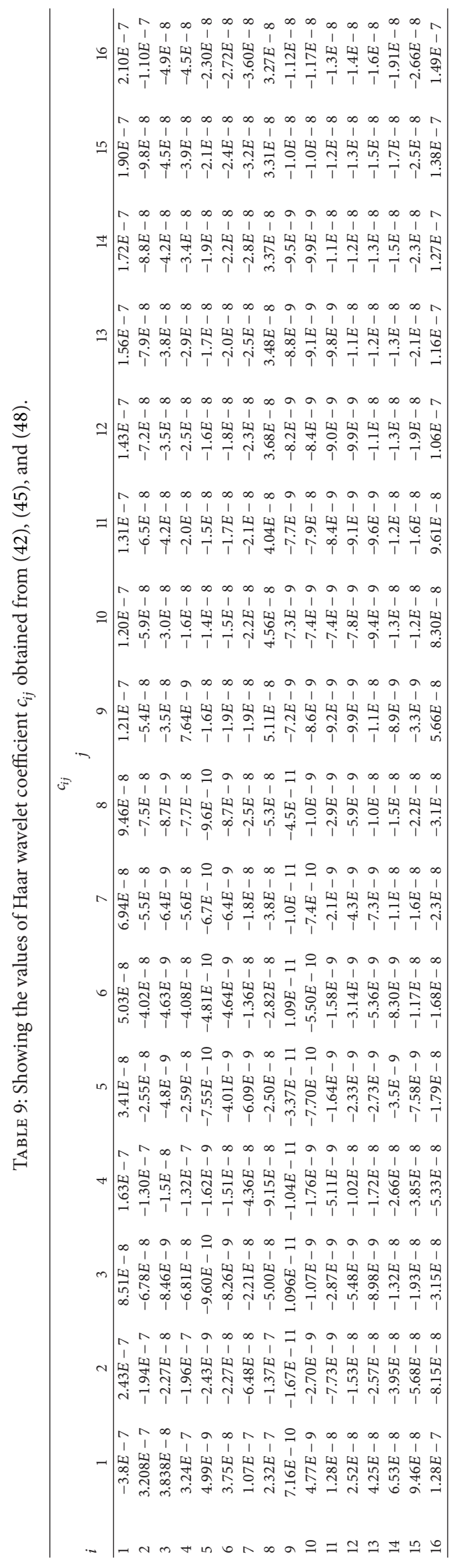




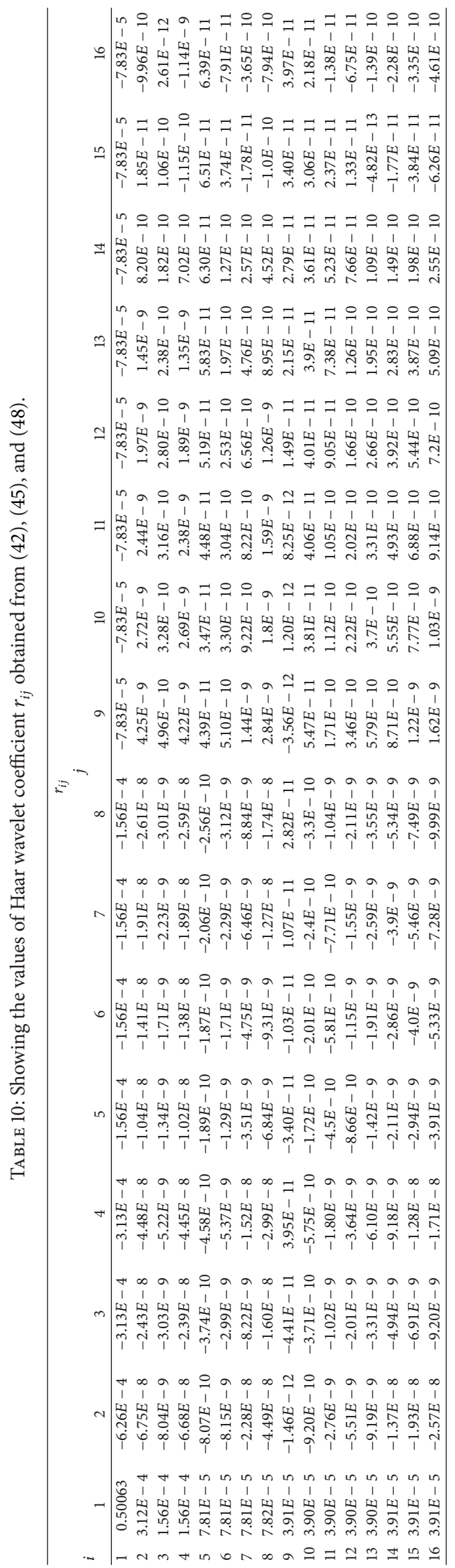




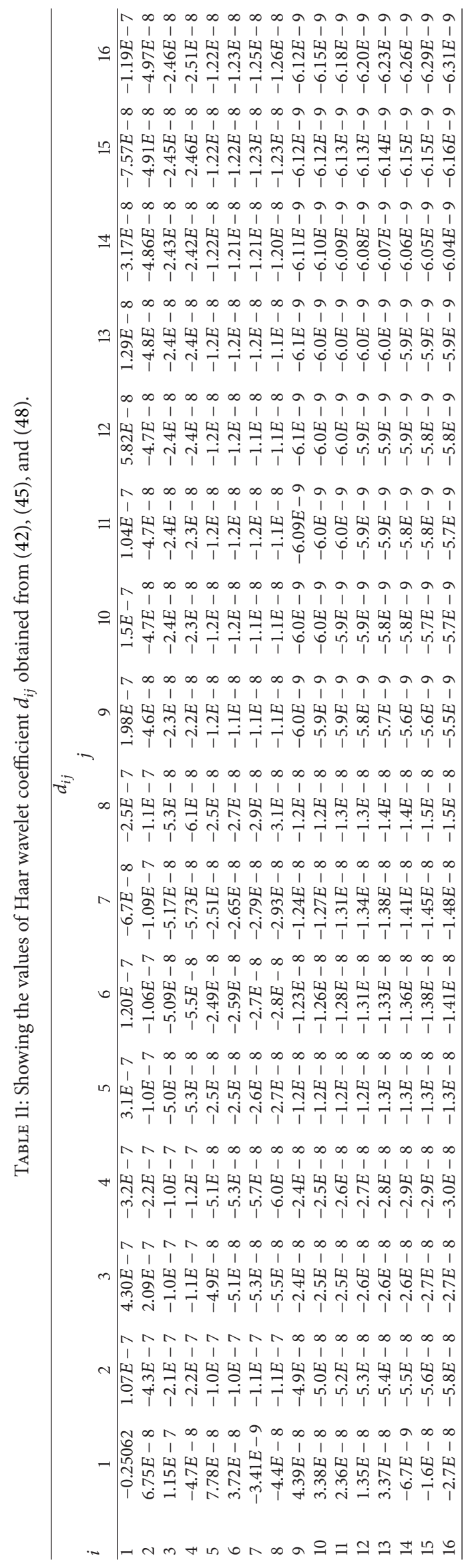


For Burgers-Fisher equation, OHAM provides more accurate results than the Haar wavelet method as shown in Table 1. But in case of generalized Fisher's equation both of the methods are competitive. The results obtained by OHAM are slightly more accurate than the results obtained by Haar wavelet method. The results obtained by OHAM are accurate as its convergence region can be easily adjusted and controlled. The main advantages of these schemes are their simplicity, applicability, and less computational errors.

\section{Conflict of Interests}

The authors declare that there is no conflict of interests regarding the publication of this paper.

\section{Acknowledgment}

This research work was financially supported by DST, Government of India, under Grant no. SR/S4/MS.:722/11.

\section{References}

[1] I. Podlubny, Fractional Differential Equations, Academic Press, New York, NY, USA, 1999.

[2] S. Saha Ray, "On Haar wavelet operational matrix of general order and its application for the numerical solution of fractional Bagley Torvik equation," Applied Mathematics and Computation, vol. 218, no. 9, pp. 5239-5248, 2012.

[3] S. Saha Ray and A. Patra, "Haar wavelet operational methods for the numerical solutions of fractional order nonlinear oscillatory Van der Pol system," Applied Mathematics and Computation, vol. 220, pp. 659-667, 2013.

[4] C. F. Chen and C. H. Hsiao, "Haar wavelet method for solving lumped and distributed parameter-systems," IEE ProceedingsControl Theory and Applications, vol. 144, no. 1, pp. 87-94, 1997.

[5] V. Marinca, N. Herişanu, C. Bota, and B. Marinca, "An optimal homotopy asymptotic method applied to the steady flow of a fourth-grade fluid past a porous plate," Applied Mathematics Letters, vol. 22, no. 2, pp. 245-251, 2009.

[6] R. A. Shah, S. Islam, A. M. Siddiqui, and T. Haroon, "Optimal homotopy asymptotic method solution of unsteady second grade fluid in wire coating analysis," Journal of the Korean Society for Industrial and Applied Mathematics, vol. 15, no. 3, pp. 201-222, 2011.

[7] M. Ghoreishi, A. I. B. Md. Ismail, A. K. Alomari, and A. S. Bataineh, "The comparison between homotopy analysis method and optimal homotopy asymptotic method for nonlinear age-structured population models," Communications in Nonlinear Science and Numerical Simulation, vol. 17, no. 3, pp. 1163-1177, 2012.

[8] J. Zhang and G. Yan, "A lattice Boltzmann model for the Burgers-Fisher equation," Chaos, vol. 20, no. 2, pp. 1-12, 2010.

[9] Y. Liu, "General solution of space fractional Fisher's nonlinear diffusion equation," Journal of Fractional Calculus and Applications, vol. 1, no. 2, pp. 1-8, 2011.

[10] A. M. Wazwaz and A. Gorguis, "An analytic study of Fisher's equation by using Adomian decomposition method," Applied Mathematics and Computation, vol. 154, no. 3, pp. 609-620, 2004. 


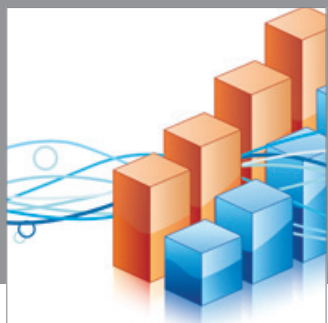

Advances in

Operations Research

mansans

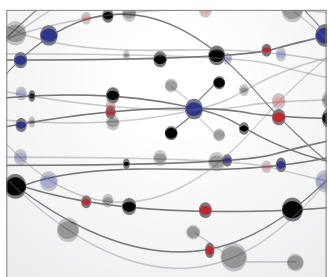

The Scientific World Journal
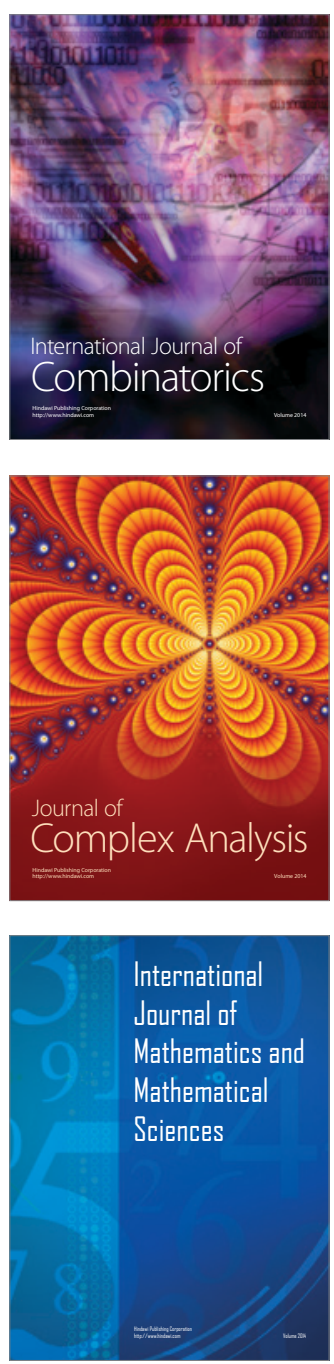
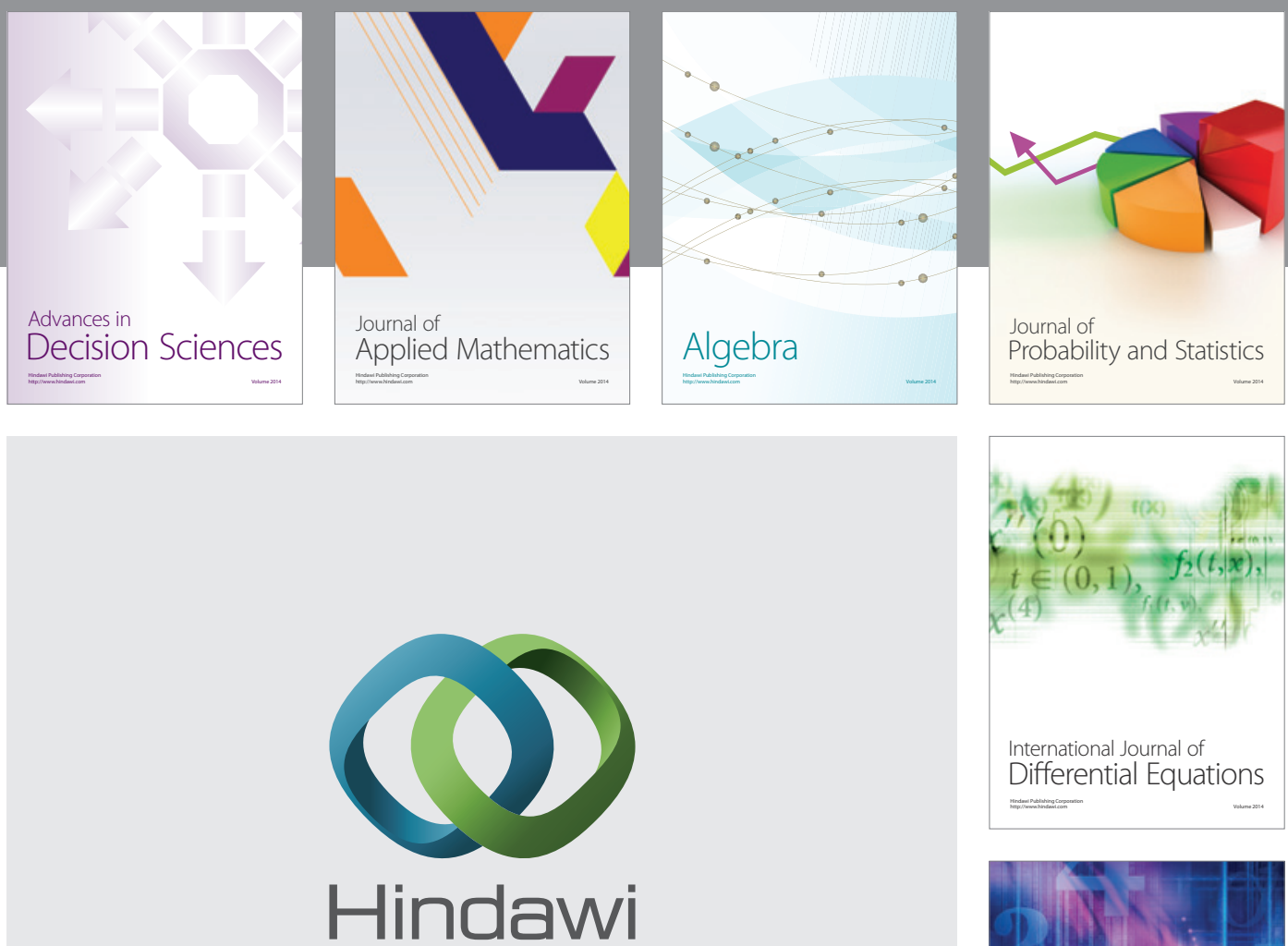

Submit your manuscripts at http://www.hindawi.com
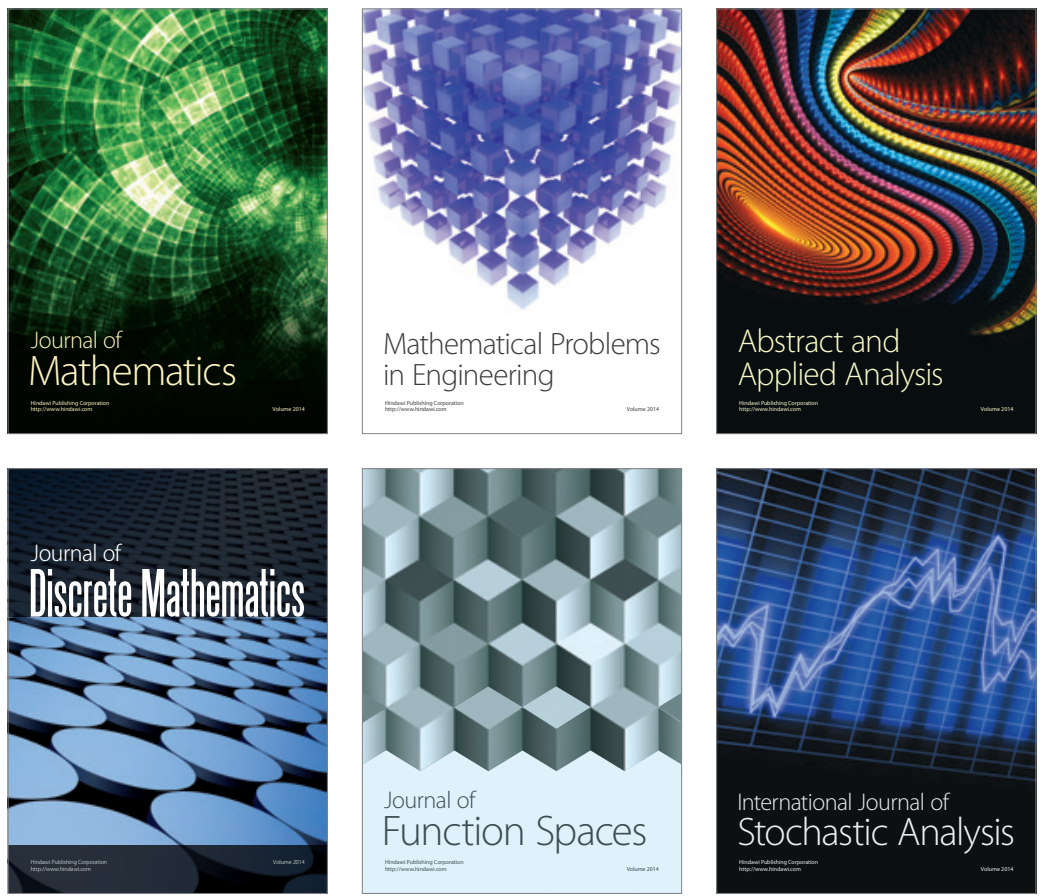

Journal of

Function Spaces

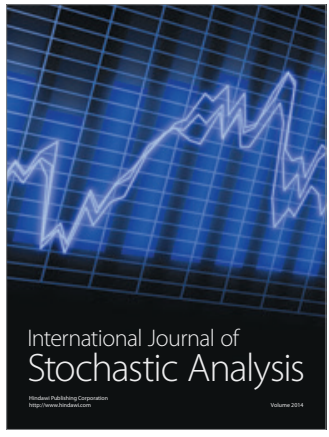

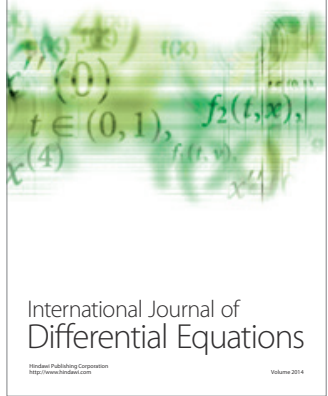
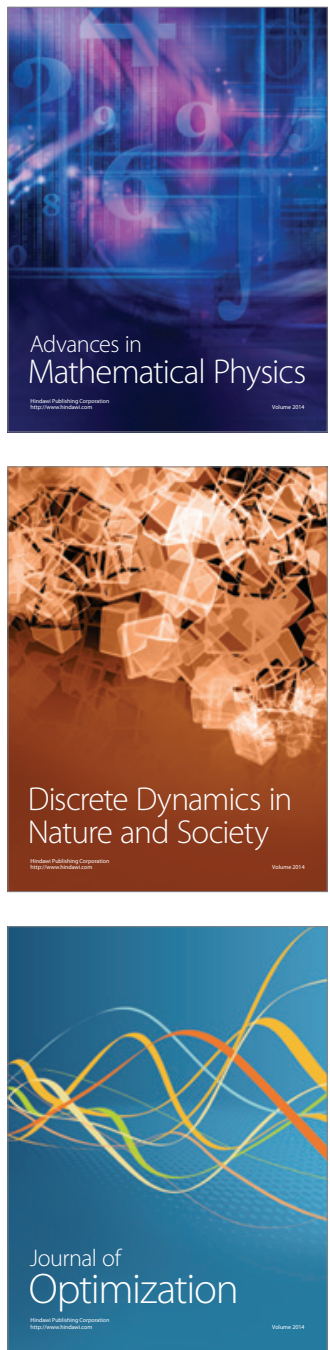